Title: BEAM MATCHING AND HALO CONTROL

$$
\operatorname{CON}-970503--262
$$

\section{Author(s): Walter Lysenko Zohreh Parsa}

\section{LANSCE-1}

Brookhaven Nat'l

Laboratory

\section{RECENDO \\ AUG 141937 \\ OSTI}

Submitted To: 1997 Particle Accelerator Conference Vancouver, BC, Canada

May 12-16, 1997
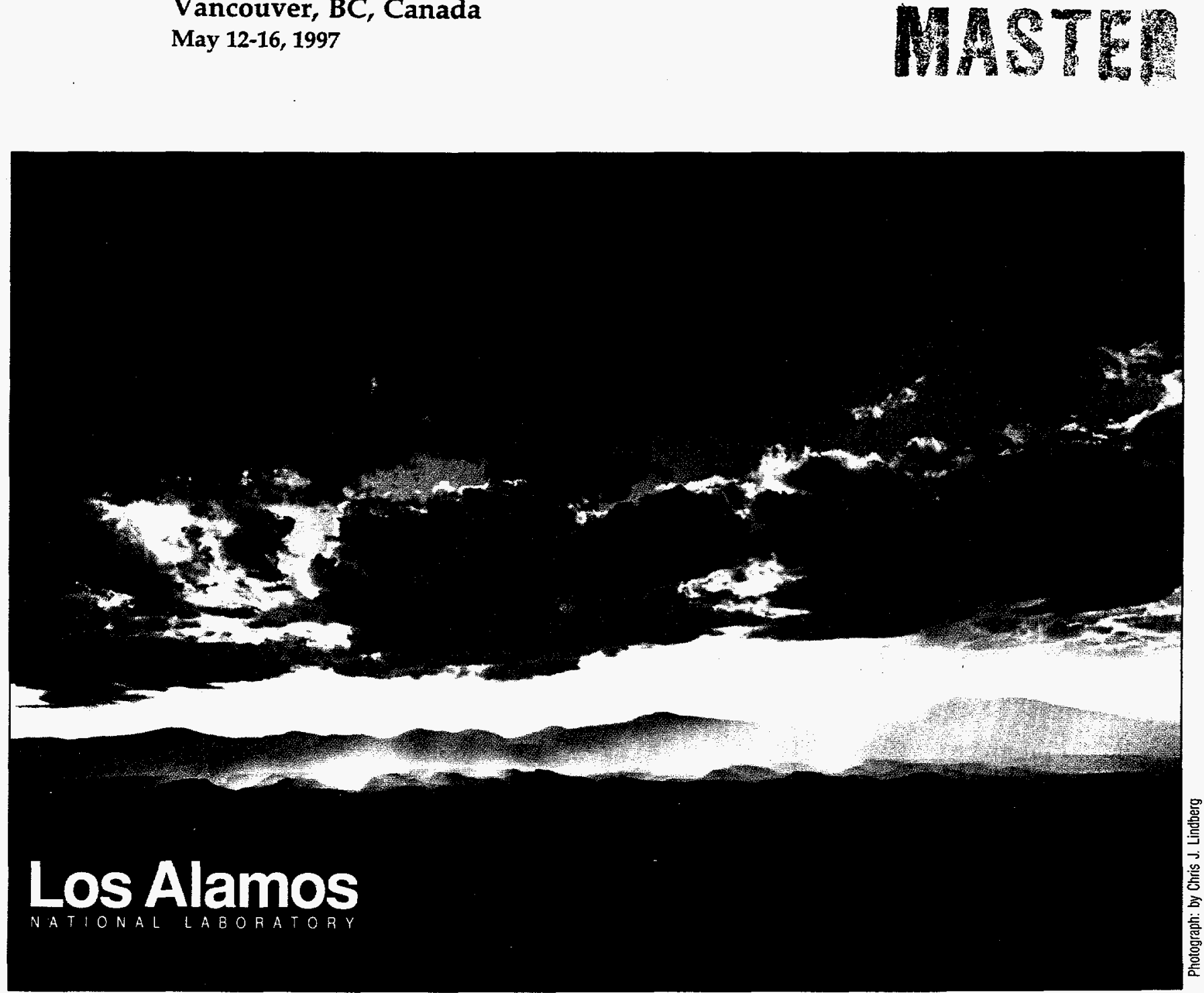

This is a preprint of a paper intended for publication in a journal or proceedings. Because changes may be made before publication, this preprint is made available with the understanding that it will not be ciled or reproduced without the permission of the author. 


\title{
BEAM MATCHING AND HALO CONTROL
}

\author{
W. Lysenko, Los Alamos National Laboratory, MS H808, Los Alamos, NM 87545, USA and \\ Z. Parsa, Brookhaven National Laboratory, 901A Physics Dept., Upton, NY 11973-5000, USA
}

\section{Abstract}

We present an overview of the status of ongoing work on physics models describing beam matching and halo control for particle accelerators, particularly high power ion linacs. We consider moments and various new variables that more naturally describe beam halo evolution. We compute matched beams and "mode invariants" (analogs of moment invariants) using primarily symbolic techniques.

\section{INTRODUCTION}

Future intense ion linacs will require good control of beam halo. To understand halo production, we consider the evolution of the phase-space distribution in the Vlasov regime. Since this is a non-imaging application, single-particle motion and its associated concepts such as aberrations are not as relevant. What is important is matching the beam to high order, taking into account nonlinearities such as those that arise from space charge.

Moments are an example of coordinates for the phasespace distribution. We can compute the values of the moments at any time from their initial values by solving the moment-evolution equations. The idea here is that we directly solve for the quantities of interest (properties of the distribution) rather than looking at the single-particle motion. Our goal is to extend this idea to computing the evolution of the halo, i.e., we are seeking "halo variables."

\section{MATCHING}

A matched beam is one whose distribution function $f(x, p, t)$ is a function of single-particle invariants. For a linear periodic lattice, for example, $f$ is matched if it is an arbitrary function of the Courant-Snyder invariant ellipse

$$
f(x, p, t)=F\left(\gamma x^{2}+2 \alpha x p+\beta p^{2}\right) .
$$

Any matched $f$ will be periodic in time, as will be its moments. If we see that a moment does not have the same value at times one lattice period apart, we know the beam is not matched. This is not true for the single-particle motion, which always contains a betatron-frequency component, even for a matched beam. This is the advantage of using moments or other coordinates of the distribution. For nonlinear motion, we still have periodic moments, even though the single-particle motion is more complicated.

\section{HALO VARIABLES}

An ideal set of variables would be one that includes a variable that describes the fraction of the beam outside a given radius. Knowing the evolution equation for such a variable would allow us to compute and be able to control particle loss. How close can we come to this goal?

Here, we will begin to answer this question by considering several different descriptions of beam distributions. We will compute matched beams for a simple nonlinear example and, where possible, mode invariants (the analog of moment invariants) in the new variables. One question we would like to answer is why we have not been able to determine the moment invariants for nonlinear motion.

\section{LIE-POISSON FORMULATION}

We can maintain Hamiltonian structure in distribution coordinates as follows ${ }^{1}$. Suppose we describe the solution to the Vlasov equation with some dynamical variables $G_{i}$. Think of the $G_{i}$ as functionals that map the phase-space distribution function $f(x, p, t)$ to numbers (the moments, e.g., in a moments description). The variables $G_{i}$ will form a Lie algebra if we define a Lie-Poisson (LP) bracket in terms of the ordinary Poisson bracket by

$$
\left[G_{i}, G_{j}\right]_{L P}=\iint d x d p f(x, p, t)\left[\frac{\delta G_{i}}{\delta f}, \frac{\delta G_{j}}{\delta f}\right],
$$

where $\delta G / \delta f$ is the variational derivative of $G$ with respect to $f$. The dynamics (evolution of the variables) is given by

$$
\frac{d}{d t} G=\left[G, H_{\mathrm{LP}}\right]_{\mathrm{LP}}
$$

The LP Hamiltonian $H_{\mathrm{LP}}$ is derived from the usual singleparticle Hamiltonian $H$ by

$$
\frac{\delta H_{\mathrm{LP}}}{\delta f}=H \text {. }
$$

If the functional $G$ is an integral over phase space involving $f, x, p$, and $t$, then the variational derivative is the partial derivative of the integrand with respect to $f$. In this case,

$$
H_{\mathrm{LP}}=\iint d x d p f(x, p, t) H(x, p, t)
$$

\section{EXAMPLES OF NEW VARIABLES IN LIE-POISSON FORMULATION}

We compute matched beams and mode invariants symbolically using Mathematica[1] for three kinds of variables: moments, Fourier modes, and what we call histogram modes. We define the properties of the LP bracket

\footnotetext{
${ }^{1}$ We thank D. Holm for pointing out this approach (private communication, 1989).
} 
and compute a LP Hamiltonian, which then allows us to compute the time derivatives of a quantity by taking the bracket of the quantity with the $H_{\mathrm{LP}}$. In these examples, we consider the following single-particle Hamiltonian

$$
H(x, p)=\frac{p^{2}}{2}+k \frac{x^{2}}{2}+k_{2} \frac{x^{3}}{3} .
$$

We assume the force constants $k$ and $k_{2}$ are timeindependent, which means matched beams are those whose distributions are constant in time. We compute matches by requiring the LP bracket of all variables with $H_{\mathrm{LP}}$ to be zero. To compute mode invariants, we assume a function of the variables and solve for parameters of the function that result in making the function time independent.

\subsection{Moments}

Moments are averages of monomials in phase space over the phase-space distribution. For example, $\left\langle x^{2}\right\rangle$ is defined for continuous and discrete distributions by

$$
<x^{2}>=\iint d x d p f(x, p, t) x^{2}=\frac{1}{N} \sum_{i=1}^{N} x_{i}^{2} .
$$

The LP bracket for moments is given by

$$
\left[<x^{i} p^{j}\right\rangle,\left\langle x^{m} p^{n}>\right]_{L P}=(i n-j m)<x^{i+m-1} p^{j+n-1}>\text {. }
$$

We achieve closure for finite order by replacing the above right hand side with zero if it involves moments of orders greater than the cutoff value. Note that is hard to reconstruct $f$ from the moments. Fortunately, this is not necessary to compute the LP Hamiltonian, which is simply

$$
H_{L P}=\frac{\left.<p^{2}\right\rangle}{2}+k \frac{\left.<x^{2}\right\rangle}{2}+k_{2} \frac{\left.<x^{3}\right\rangle}{3} .
$$

\subsubsection{Matched moments}

A matched beam satisfies the following relations.

$$
\begin{array}{ll}
<p^{2}>=k<x^{2}>-\frac{5 k_{2}^{2}}{k^{2}}<x^{2} p^{2}> & <p^{3}>=0 \\
<x p>=0 & <x^{4}>=\frac{3<x^{2} p^{2} \geq}{k} \\
<x^{3}>=-\frac{5 k_{2}}{k^{2}}<x^{2} p^{2}> & <x^{3} p>=0 \\
<x^{2} p>=0 & <x p^{3}>=0 \\
<x p^{2}>=-\frac{k_{2}}{k}<x^{2} p^{2}> & <p^{4}>=3 k<x^{2} p^{2}>
\end{array}
$$

Any set of moments related this way will be constant in time. The nonlinearity give rises to a nonzero value of some moments that are of odd degree in $x$. The matched distribution does not have elliptical symmetry if $k_{2} \neq 0$.

\subsubsection{Moment invariants}

Moment invariants are functions of moments that are conserved. With the nonlinearity turned off $\left(k_{2}=0\right)$, we find the invariant $I_{22}=\left\langle x^{2}\right\rangle\left\langle p^{2}\right\rangle-\langle x p\rangle^{2}$, which is just the square of the rms emittance, and higher-order invariants like $I_{44}=\left\langle x^{4}\right\rangle\left\langle p^{4}\right\rangle-4\left\langle x^{3} p\right\rangle\left\langle x p^{3}\right\rangle+$ $3<x^{2} p^{2}>^{2}$, which we already know about (see the review in Ref.[2]). Our code finds the complete set of functionallyindependent invariants, including those of mixed order (e.g., containing both second and fourth moments). Moment invariants for nonlinear motion are still unknown.

\subsection{Fourier Modes}

Define Fourier modes on a finite region of phase space by

$$
f_{m n}=\frac{1}{(2 \pi)^{2}} \int_{-\pi}^{\pi} d x \int_{-\pi}^{\pi} d p f(x, p) e^{-i(m x+n p)}
$$

The LP bracket of two of these quantities is

$$
\left[f_{i j}, f_{m n}\right]_{\mathrm{LP}}=-\frac{1}{(2 \pi)^{2}}(i n-j m) f_{i+m, j+n} .
$$

We attempt closure by zeroing higher modes. Unlike the situation for moments, this does not preserve all the properties of a Lie algebra for the truncated system, but we proceed anyway, in the hope it will lead to something useful. The LP Hamiltonian is expressed in terms of the modes by

$$
f(x, p, t)=\sum_{m, m=-N}^{N} f_{m n} e^{i(m x+n p)}
$$

\subsubsection{Matched Fourier modes}

For a cutoff of $N=1$, the matched modes satisfy

$$
\begin{array}{ll}
f_{-1,0}=(k+\bar{k}) f_{0,-1} & f_{-1,-1}=\frac{k+\bar{k}}{k-\bar{k}} f_{1,1} \\
f_{1,0}=(k-\bar{k}) f_{0,-1} & f_{-1,1}=\frac{k+\bar{k}}{k-\bar{k}} f_{1,1} \\
f_{0,1}=f_{0,-1} & f_{1,-1}=f_{1,1}
\end{array}
$$

where $\bar{k}=i k_{2}\left(\pi^{2}-6\right) / 3$. There are nine modes at this order, so there are three free modes for a matched beam.

\subsubsection{Fourier mode invariants}

For $k_{2}=0$, some invariants (truncated at order 1) are

$$
\begin{aligned}
& f_{0,0}=\text { const. } \\
& f_{-1,-1}+f_{-1,1}+f_{1,-1}+f_{1,1}=\text { const. }
\end{aligned}
$$

The first of these is just the conservation of particles and is equivalent to $<1>=$ const. for moments.

\subsection{Histogram Variables}

Let us divide phase space into rectangular bins labeled by the indices $m$ in the $x$-direction and $n$ in the $y$-direction. Let both indices range from $-N$ to $N\left(4 N^{2}\right.$ bins total). Let the mode $f_{m n}$ describe the density in phase space at the bin specified by the indices $m$ and $n$. The following 
LP bracket will approximate the correct physics (again, the truncated system is not exactly Hamiltonian).

$$
\left[f_{i j}, f_{m n}\right]_{\mathrm{LP}}=\left\{\begin{array}{c}
(m-i)(n-j)\left(f_{i n}-f_{m j}\right), \\
|m-i|=1 \text { and }|n-j|=1 \\
0, \quad \text { otherwise. }
\end{array}\right.
$$

Note that closure for these variables is a boundary problem. If the beam is localized (as are real beams) then closure is automatic and involves no additional physics approximations. The LP Hamiltonian is

$$
H_{\mathrm{LP}}=\sum_{m, n=-N}^{N} f_{m n}\langle H\rangle_{m n},
$$

where $\langle H\rangle_{m n}$ is the average of $H(x, p)$ over the bin.

\subsubsection{Matched histogram modes}

In the continuous case, a matched beam would be elliptical for $k_{2}=0$. We find $N$ independent modes (bin values) in a matched beam. Figure 1 shows an example with and without the nonlinearity. Equal densities in phase space are depicted by equal gray levels.
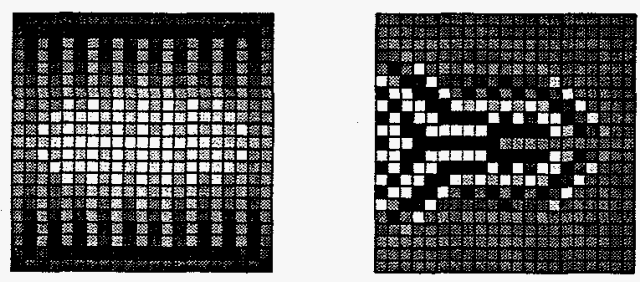

Figure 1: Matched histogram without nonlinearity (left) and with nonlinearity (right).

\subsubsection{Histogram mode invariants}

For general $k$ and $k_{2}$, we found one invariant of degree 1 in the modes. For $N$ odd it consists of the sum of all modes having odd indices and for $N$ even, it consists of the sum of all modes having even indices. For $k_{2}=0$, the mode $f_{0,0}$ is separately conserved.

\section{WEIGHTED MOMENTS}

Consider generalizing the moments, not by using a new set of basis functions, but by changing the weights. Define "weighted moments" by

$$
\begin{aligned}
<g(x, p)>_{n} & =\frac{\iint d x d p[f(x, p, t)]^{n+1} g(x, p)}{\iint d x d p[f(x, p, t)]^{n+1}} \\
& =\frac{\sum_{i=1}^{N}\left[f\left(x_{i}, p_{i}, t\right)\right]^{n} g\left(x_{i}, p_{i}\right)}{\sum_{i=1}^{N}\left[f\left(x_{i}, p_{i}, t\right)\right]^{n}}
\end{aligned}
$$

where the basis functions $g(x, p)$ are monomials in $x$ and $p$. The usual moments result when the weight index is $n=0$. For negative values of $n$, the halo is emphasized. Numerical experiments indicate that it is possible to get the Courant-Snyder parameters for the halo by using weighted moments with weight indices of about $n=-2$. A LP formulation of weighted moments is difficult (part of the problem is that they are defined by a nonlinear functional) and will not be done here. A nice feature of the weighted moments is that we already know the dynamics of these objects. Since the distribution function is constant on phasespace trajectories, so is any function of it. Thus

$$
\left.\left.\frac{d}{d t}<g(x, p)\right\rangle_{n}=<\frac{d}{d t} g(x, p)\right\rangle_{n},
$$

just as for regular moments. So, we already know the linear invariants. For example, we have

$$
\left\langle x^{2}\right\rangle_{n}\left\langle p^{2}\right\rangle_{n}-\langle x p\rangle_{n}^{2}=\text { const. }
$$

which is the weighted-moment analog of rms emittance.

Instead of using higher-order moments to achieve a more accurate beam description, we can combine second-order moments of various weights. In the absence of space charge, moments of different weights do not interact; core and halo evolve independently. Space charge introduces coupling through the force constants, which depend on the spatial moments of all weights.

\section{DISCUSSION}

Histogram variables are probably not the correct approach but could be usefully further studied because they so completely separate the halo and core of the beam. Weighted moments appear promising because they factor the core and halo motion in a very desirable way. They can give us the evolution of the Courant-Snyder parameters for the core and halo separately; there is no need to go to higher moments to see the halo. We need not consider higher moments unless the additional physics they represent is actually involved in halo generation. Another advantage of this approach is that it could be used to extend existing codes like TRACE 3-D[3] to include nonlinear effects.

\section{ACKNOWLEDGEMENTS}

We thank Paul Channell for helpful comments. This work was partially carried out at the Institute for Theoretical Physics, University of California, Santa Barbara, as part of the New Ideas for Particle Accelerators Program with partial support by the U.S. Department of Energy and the National Science Foundation.

\section{REFERENCES}

[1] Stephen Wolfram, The Mathematica Book, 3rd ed. (Wolfram Media/Cambridge University Press, 1996).

[2] W.P. Lysenko, "The moment approach to high-order accelerator beam optics," Nucl. Instr. and Meth. in Phys. Res. A 363 (1995) 90-99.

[3] D.P. Rusthoi, W.P. Lysenko, and K.R. Crandall, "Further improvements in TRACE 3-D," (this conference). 


\section{DISCLAIMER}

This report was prepared as an account of work sponsored by an agency of the United States Government. Neither the United States Government nor any agency thereof, nor any of their employees, make any warranty, express or implied, or assumes any legal liability or responsibility for the accuracy, completeness, or usefulness of any information, apparatus, product, or process disclosed, or represents that its use would not infringe privately owned rights. Reference herein to any specific commercial product, process, or service by trade name, trademark, manufacturer, or otherwise does not necessarily constitute or imply its endorsement, recommendation, or favoring by the United States Government or any agency thereof. The views and opinions of authors expressed herein do not necessarily state or reflect those of the United States Government or any agency thereof. 


\section{DISCLAMMIER}

Portions of this document may be illegible in electronic image products. Images are produced from the best available original document. 